\title{
Familial hypercholesterolaemia as an example of early diagnosis of coronary artery disease risk by DNA techniques
}

\author{
SE HUMPHRIES \\ From the Charing Cross Sunley Research Centre, Hammersmith, London
}

There are many families in which several individuals have a history of early heart attack. This implies that in such families a major genetic component contributes to the risk of developing ischaemic heart disease. Molecular biology techniques may allow the identification of individuals who are at high risk of developing ischaemic heart disease because they have this inherited component. Such individuals could be monitored closely and advised on diet and life style or possibly offered drug treatment to reduce their subsequent risk of early heart attack.

When will cardiologists be able to ask for a certain gene probe test to be carried out on a patient before they decide on what advice to give the patient and his family on prognosis, or the treatment to be offered, or even the diagnosis? At the current rate of progress useful tests may become available within the next few years. At present the best example of how these tests can be usefully applied to patients with atherosclerosis is in the presymptomatic diagnosis of familial hypercholesterolaemia.

\section{Familial hypercholesterolaemia analysis and diagnosis at the gene level}

Cholesterol, with its associated carrier proteins, is transported in the circulation in different classes of lipoprotein. ${ }^{12}$ Epidemiological studies have shown that high concentrations of one of these cholesterol rich lipoproteins, low density lipoprotein (LDL), are associated with increased risk of atherosclerosis and myocardial infarction. ${ }^{3}$ LDL is normally

Requests for reprints to Dr S E Humphries, The Charing Cross Sunley Research Centre, Lurgan Avenue, Hammersmith, London W6 8LW. removed by a specific LDL receptor found on all cells, including the liver. In familial hypercholesterolaemia there is a defect in this receptor. ${ }^{4-6}$ Individuals with one normal receptor gene and one defective receptor gene have roughly twice the normal concentration of serum LDL and an increased rate of atherosclerosis and heart attacks in mid-life.

A method of detecting the LDL receptor gene is the first requirement for identifying individuals at risk of familial hypercholesterolaemia. A gene specific probe that is a fragment of the normal receptor gene can be isolated by molecular biology techniques. This piece of DNA is "immortalised" by inserting it into a bacterial plasmid, a circle of DNA which can multiply inside a bacterium. A large amount of the plasmid containing the fragment of the LDL receptor gene can be prepared. Such a probe for the human LDL receptor gene was isolated by Brown and Goldstein and their coworkers in $1984 .^{7}$ They have generously made this probe freely available for research purposes and it has been used by them and others to study the defects occurring in familial hypercholesterolaemia ${ }^{89}$ and to develop methods of diagnosis. ${ }^{10}$

A technique developed by $\mathrm{Dr}$ Ed Southern in Edinburgh, and named after him-the "Southern blot"-is used to detect the LDL receptor gene in the DNA. ${ }^{11}$ DNA from a patient can be isolated from the leucocytes in a small sample of peripheral venous blood. This DNA is then incubated with a restriction enzyme. ${ }^{12}$ These enzymes are purified from bacteria, and they recognise and cleave DNA at specific sequences of four, five, or six bases. The DNA will be cut into many millions of specific fragments, one or more of which will contain the LDL receptor gene. These fragments are separated 


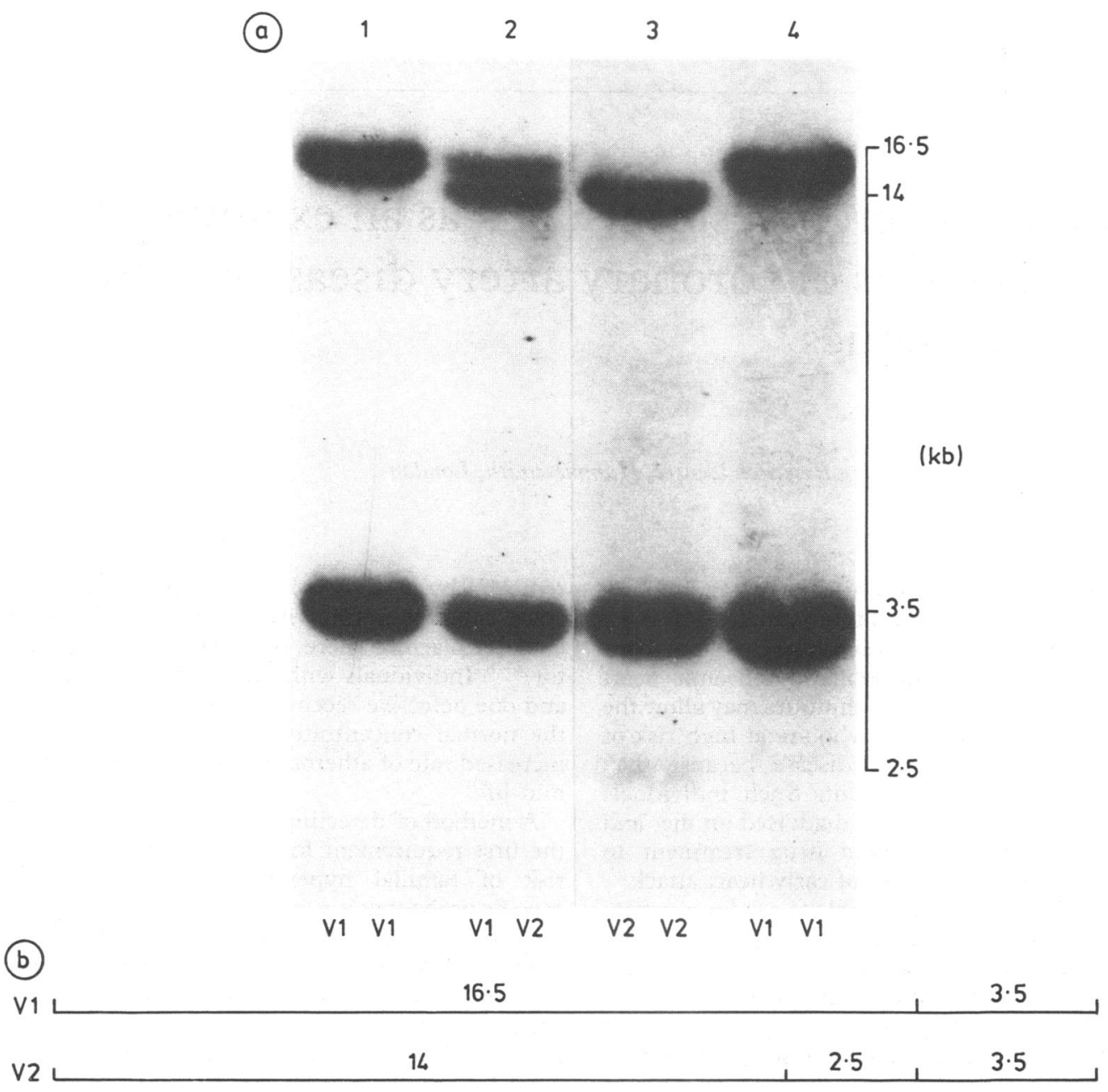
(kb) (kb)

Fig 1 Autoradiograph of a Southern blot. (a) DNA polymorphism detected with the LDL receptor gene probe. Southern blot analysis of hybridisation pattern obtained from a PvuII digest of DNA from four individuals, ${ }^{10}(b)$ Diagram of how DNA fragments detected by means of the probe can be produced by the presence of an extra PvuII site within the sequence of the gene. Cutting sites for PvuII are indicated.

according to size by electrophoresis in an agarose gel. The normally double stranded DNA is made single stranded by soaking the gel in alkali, and the DNA is transferred to a sheet of nitrocellulose filter or nylon membrane.

The next step is to make the DNA probe highly radioactive. It can then be used to detect the normal or defective LDL receptor genes present on the membrane. The membrane is incubated with the radioactive probe, which finds the matching single stranded LDL receptor gene fragments and "hybridises" with them thus reforming the DNA double helix. This process is highly specific and the probe does not hybridise with any of the other millions of gene fragments on the membrane. Radioactive probe that has not hybridised is washed off and an $x$ ray film is exposed to the membrane. On the resulting autoradiogram only the two LDL receptor genes are detected as black bands because the radioactive probe has bound to them specifically.

The two LDL receptor genes in an individual can be distinguished through the choice of restriction enzyme used for cleavage. The chromosomes, from which the two fragments are derived, have the bases 
(a)

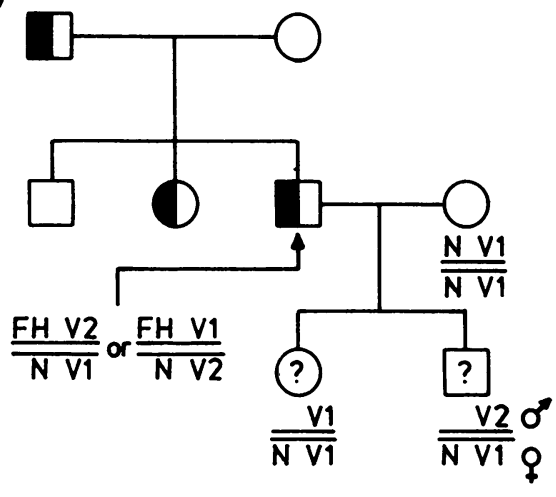

(b)

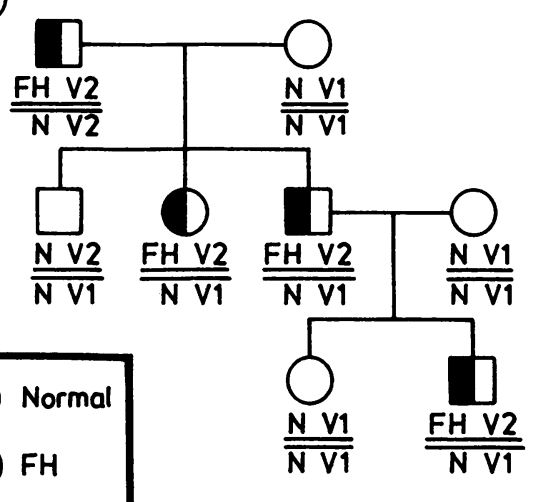

Fig 2 Segregation of familial hypercholesterolaemia and the LDL receptor gene polymorphism in a model family. $N$, normal LDL gene; FH, familial hypercholesterolaemia gene.

making up the DNA in a slightly different order. This is a consequence of slow evolutionary processes: most of these differences are "neutral" and have no pathological importance. By chance, however, they may create or destroy a cleavage site for a particular restriction enzyme. This change will be inherited and will alter the size of the fragments detected in the Southern blot procedure. This enables us to distinguish the two alleles of the LDL receptor gene in an individual and can be used for diagnosis. The technique takes advantage of the existence of a polymorphism at the DNA level. By analogy, the $\mathrm{ABO}$ blood group system is an example of polymorphism at the protein level.

Figure 1 shows a common DNA polymorphism of the LDL receptor gene. The DNA has been digested with the restriction enzyme PvuII and several fragments of the receptor gene have been detected. The DNA from all individuals contains a fragment of about 3500 base pairs (or 3.5 kilobases (kb); 1000 base pairs $=1 \mathrm{~kb}$ ). Different individuals show one of three patterns. They may have a single large fragment of about $16.5 \mathrm{~kb}$, designated the $V 1$ allele of the gene, or two fragments of $14 \mathrm{~kb}$ and 2.5 $\mathrm{kb}$, designated the $V 2$ allele. Some individuals have all the fragments, and are designated V1.V2. In these individuals we can therefore distinguish the two "alleles" of the LDL receptor gene and can potentially carry out a diagnosis. ${ }^{10}$

Figure 2 shows a family in which early diagnosis might be useful. The father had had an early myocardial infarction and is hypercholesterolaemic. His father and sister are also hypercholesterolaemic, and it is clear that this is a family with familial hypercholesterolaemia. His two children are under 10 years old, and their concentrations of LDL choles- terol are both in the 70th to 80th percentile for their age. Has either of them inherited the defective LDL receptor and will they therefore be at risk of developing premature atherosclerosis? The DNA analysis gives the results shown in fig 2a. In the father we can distinguish the two alleles of the LDL receptor gene, but we cannot tell whether the defective gene is on the chromosome with the $V 2$ allele or that with the $V 1$ allele. The mother has the genotype $V 1 V 1$ and must pass on a normal LDL receptor gene with the $V 1$ allele to both children. The daughter has therefore inherited the $V 1$ allele from the father and the son has inherited the $V 2$ allele. This must mean that one of them will have inherited the defective gene and one the normal gene.

Analysis of DNA samples from the grandparents will resolve this problem (fig $2 b$ ). The affected grandfather has the genotype $V 2 V 2$, and the normal grandmother has $V 1 V 1$. This means the familial hypercholesterolaemia gene must have been inherited with the $V 2$ allele from the grandfather. It is likely that the grandfather will already be dead of an early heart attack. In this situation we can still carry out the diagnosis based on a sample from the grandmother, because we can safely infer that the defective gene and the $V 2$ allele have been inherited from the grandfather. If both grandparents are dead it may still be possible to carry out diagnosis with information from the aunts and uncles and their children, though this may not always give a definitive answer. In this family it is clear that the daughter has inherited the normal LDL receptor gene with the $V 1$ allele and the son the defective gene with the $V 2$ allele. He will therefore develop hypercholesterolaemia, and this can be monitored, and he can be given advice on life style, diet, and 
possible drug treatment, such as cholestyramine, ${ }^{13}$ if appropriate.

There are several points to be made about this sort of analysis. In this family the defective LDL receptor gene was found to be on the chromosome with the extra PvuII site (the V2 allele). A priori, however, there is no way of telling whether the defective gene is on the chromosome with this site, or the one without it. The altered site itself is not within the part of the gene that contains information coding for the protein, and does not itself cause the defect. In fact, over $30 \%$ of both normal individuals and people with familial hypercholesterolaemia have the genotype V1V2. This means we cannot use this method to screen the population, and other techniques will have to be developed. The other immediate problem is that many patients are either $V 1 V 1$ or $V 2 V 2$, and we cannot use this polymorphism to distinguish their normal and defective LDL receptor gene alleles. In order to carry out diagnosis in these individuals, other polymorphisms must be found, and a great deal of research effort is currently being put into this.

\section{Prospects}

The gene for the LDL receptor is only one of the many genes that must be involved in the development of hyperlipidaemia. Although about 1 in 500 members of the population have familial hyperlipaemia, or about 100000 people in the United Kingdom, the defect probably accounts for $<10 \%$ of all early deaths from heart attack. It is, however, a very good example of how defects in a single gene may increase risk of early arterial disease, and how DNA tests can detect family members who have inherited the defective gene. DNA probes for other genes, for example for apolipoproteins or other receptors, are already available and more will be developed in the next few years. Many of these, taken together as a battery of tests, may be useful for the early diagnosis of hyperlipidaemia and atherosclerosis.

Just as there are genes involved in determining the serum LDL concentration in an individual, there must be genes involved in determining the response, for example, of the cells of the artery wall to this LDL. At present, we do not know which genes are involved. One cell that has an important role in the development of the atherosclerotic lesion is the macrophage. ${ }^{14} \mathrm{~A}$ study of the genes for the receptors, enzymes, and growth factors produced in these cells would be interesting and might lead to further diagnostic tools. By extension of this argument, there must also be genetic control of the processes of thrombosis ${ }^{1516}$ such as clot formation, lysis, and platelet aggregation. In addition, there may be inherited defects in some of the structural components of the heart or blood vessel walls that may predispose to cardiovascular disease in later life. For instance, subtle defects in a cardiac specific collagen in the heart valves may be causing some forms of mitral valve prolapse ${ }^{17}$ and this too would be amenable to these methods of analysis.

It therefore seems likely that molecular biology will become a useful tool for the cardiologist. The challenge for the next few years is to identify the genes and the mutations of these genes that are involved in determining an individual's risk for developing coronary artery disease. For the patient presenting with myocardial infarction, conventional management methods may remain paramount. The new techniques, however, may allow a better understanding of the particular genetic factors responsible for the patient's existing coronary artery disease. This knowledge will improve the prognosis of the patient, who can be treated more appropriately, and will allow early diagnosis of other family members who also have a genetic predisposition to atherosclerosis. It is hoped that this information will make a useful contribution to an overall strategy to reduce an individual's risk of coronary artery disease.

This work was supported by the British Heart Foundation and the Charing Cross Sunley Research Trust. I thank Dr Anna Kessling for useful discussion.

\section{References}

1 Mahley RW, Innerarity TL, Rall SC, Weisgraber KH. Plasma lipoproteins: apolipoprotein structure and function. $\mathcal{F}$ Lipid Res 1984;25:1277-94.

2 Thompson G. Apoproteins: determinants of lipoprotein metabolism and indices of coronary risk. $\mathrm{Br}$ Heart f 1984;51:585-8.

3 Keys A, Aravanis C, Blackburn $\mathrm{H}$, et al. Probability of middle-aged men developing coronary heart disease in five years. Circulation 1972;45:815-28.

4 Brown MS, Goldstein JL. Familial hypercholesterolaemia. In: Stanbury JB, Wyngaarden JB, Frederickson DS, Goldstein JL, Brown MS, eds. The metabolic basis of inherited human disease. New York: Macgraw Hill, 1983:500-50.

5 Brown MS, Goldstein JL. How LDL receptors influence cholesterol and atherosclerosis. Sci $\mathrm{Am}$ 1984;251:52-60.

6 Tolleshaug H, Hobgood KK, Brown MS, Goldstein JL. The LDL receptor locus in familial hypercholesterolaemia. Multiple mutations disrupt transport and processing of a membrane receptor. Cell 1983;32:941-51.

7 Yamamoto T, Davis LG, Brown MS, et al. The human LDL receptor: a cysteine-rich protein with multiple 
Alu sequences in its mRNA. Cell 1984;39:27-38.

8 Lehrman MA, Schneider WJ, Sudhof TC, Brown MS, Goldstein JL, Russell DW. Mutations in LDL receptor; Alu-Alu recombination deletes exons encoding transmembrane and cytoplasmic domains. Science 1985;227:140-6.

9 Horsthemke B, Kessling AM, Seed M, Wynn V, Williamson $R$, Humphries SE. Identification of a deletion in the low density lipoprotein (LDL) receptor gene in a patient with familial hypercholesterolaemia. Hum Genet 1985;71:75-8.

10 Humphries SE, Kessling AM, Horsthemke B, et al. A common DNA polymorphism of the low density lipoprotein (LDL) receptor gene and its use in diagnosis. Lancet 1985;i:1003-5.

11 Southern E. Detection of specific sequences among DNA fragments separated by gel electrophoresis. f Mol Biol 1975;98:503-17.

12 Malcolm ADB. The use of restriction enzymes in genetic engineering. In: Williamson $\mathbf{R}$, ed. Genetic Engineering. Vol 2. London: Academic Press,
1981:129-73.

13 Lipid Research Clinics Program. The Lipid Research Clinics coronary primary prevention trial. Results II. The relationship of reduction in incidence of coronary heart disease to cholesterol lowering. $\mathcal{F} A M A$ 1984;251:365-74.

14 Brown MS, Goldstein JL. Lipoprotein metabolism in the macrophage: implications for cholesterol deposition in atherosclerosis. Annu Rev Biochem 1983;52: 223-61.

15 Mead TW, North WRS, Chakrabarty R, Stirling Y, Haines AP, Thompson SG. Haemostatic function and cardiovascular death: early results of a prospective study. Lancet 1980;i:1050-3.

16 O'Connor NTJ, Cederholm-Williams S, Copper S, Cotter L. Hypercoagulability and coronary artery disease. Br Heart $\mathcal{F}$ 1984;52:614-6.

17 Devereux RB, Brown TW, Kramer-Fox R, Sachs I. Inheritance of mitral valve prolapse: effect of age and sex on gene expression. Ann Intern Med 1982;97:826-32. 